\title{
Is there any opportunity for immune checkpoint inhibitor therapy in non-small cell lung cancer patients with brain metastases?
}

\author{
Lizza E. L. Hendriks ${ }^{1}$, Jordi Remon ${ }^{2}$, Jessica Menis ${ }^{3,4}$, Benjamin Besse ${ }^{5,6}$ \\ ${ }^{1}$ Department of Pulmonary Diseases, GROW - School for Oncology and Developmental Biology, Maastricht University Medical Center, Maastricht, \\ The Netherlands; ${ }^{2}$ Department of Medical Oncology, Centro Integral Oncológico Clara Campal (HM CIOCC), Hospital HM Delfos, HM \\ Hospitales, Barcelona, Spain; ${ }^{3}$ Department of Surgery, Oncology and Gastroenterology, University of Padova, Padova, Italy; ${ }^{4}$ Medical Oncology \\ Department, Istituto Oncologico Veneto IRCCS, Padova, Italy; ${ }^{5}$ Department of Medical Oncology, Gustave Roussy Cancer Campus, Institut \\ d'Oncologie Thoracique (IOT), Gustave Roussy, Université Paris-Saclay, Villejuif, France; ${ }^{6}$ Paris-Sud University, Orsay, France \\ Contributions: (I) Conception and design: All authors; (II) Administrative support: LEL Hendriks; (III) Provision of study materials or patients: None; \\ (IV) Collection and assembly of data: LEL Hendriks, J Remon, J Menis; (V) Data analysis and interpretation: All authors; (VI) Manuscript writing: \\ All authors; (VII) Final approval of manuscript: All authors. \\ Correspondence to: Lizza E. L. Hendriks, MD, PhD. Department of Pulmonary Diseases, Maastricht University Medical Center+, PO Box 5800,6202 \\ AZ Maastricht, The Netherlands. Email: lizza.hendriks@mumc.nl.
}

\begin{abstract}
Although brain metastases occur in almost one-third of non-small cell lung cancer (NSCLC) patients, and immune checkpoint inhibitors (ICI) either as monotherapy or combined with chemotherapy are the new standard of care in the first line setting, most trials excluded patients with asymptomatic and/ or untreated brain metastases. Brain metastases have a major clinical impact due to the worsening of the patient's prognosis and quality of life. Furthermore, the incidence of brain metastases is increasing in NSCLC patients, due to a longer survival and better imaging techniques. Therefore, brain metastases are increasingly becoming a research topic. Recent clinical data endorses ICI as a therapeutic strategy in this subpopulation of NSCLC patients, although the immune environment in brain metastases is more immune ignorant compared with the microenvironment in the primary tumour or in the extracranial metastases. In this review we summarize the current evidence of ICI strategy in NSCLC patients with brain metastases, including trial and real-life data. We also state that the different tumor microenvironment between brain metastases and primary tumor may explain the discordance on the response rate during treatment with ICI. Last, we focus on future directions, including the role and optimal sequence of cranial irradiation and ICI, prognostic scores, the best response assessment and new imaging techniques.
\end{abstract}

Keywords: Non-small cell lung cancer (NSCLC); brain metastases; immune checkpoint inhibitors

Submitted Feb 26, 2020. Accepted for publication May 13, 2020.

doi: $10.21037 /$ tlcr-20-343

View this article at: http://dx.doi.org/10.21037/tlcr-20-343

\section{Introduction}

Brain metastases remain the main cause of poor quality of life (QoL) and survival in non-small cell lung cancer (NSCLC) patients (1). Thirty to sixty percent of patients will be diagnosed with brain metastases during their disease (2-4). Treatment of these metastases is crucial for better symptom control and to improve survival. Therefore, strategies to improve outcomes in this patient population are needed. Immune checkpoint inhibitors (ICI), mainly anti programmed cell death 1 (anti-PD-1) and programmed cell death ligand-1 (anti-PD-L1), have become standard of care in the upfront therapeutic strategy of wild-type advanced NSCLC either as monotherapy or in combination with chemotherapy. This ICI strategy has changed the prognosis of advanced NSCLC: 5 -year overall survival (OS) is now ranging from $15 \%$ to $23 \%$ according to the treatment line (5). Nonetheless, due to concerns regarding 
the risk-benefit ratio, poor outcome and the risk of brain pseudoprogression, the majority of phase III trials excluded patients with asymptomatic and/or untreated brain metastases and the percentage of patients with brain metastases included in these trials was below $20 \%$ (6-8). Of note, in daily practice, almost one quart of NSCLC patients with brain metastases derive benefit from monotherapy ICI treatment (9). In general, monotherapy with ICI achieves an intracranial objective response rate (ic-ORR) that mirrors extracranial ORR $(9,10)$. This was demonstrated in a phase II study enrolling $37 \mathrm{PD}-\mathrm{L} 1$ positive NSCLC patients with untreated brain metastases with a maximum diameter of $20 \mathrm{~mm}$ : icORR was $29.7 \%$ for pembrolizumab $(10,11)$. Similarly, in a retrospective series including 73 NSCLC patients with active brain metastases, treated with anti-PD(L)1, the icORR was $27 \%$ (9). Importantly, responses can be durable, as $34 \%$ of patients in the phase II study were alive for more than two years (10).

Since both growth of brain metastases and inflammation caused by ICI can cause neurological complaints, it remains a challenge to define the optimal ICI treatment strategy along with the optimal place of local treatment in NSCLC patients with brain metastases. In this review, we summarize the current evidence, including future directions for research.

\section{Brain metastases immune environment}

Generally, in NSCLC patients, benefit with monotherapy ICI increases with higher PD-L1 expression. Therefore, PD-L1 expression level is the standard predictive biomarker for making treatment decisions in first line treatment (12). In a cohort of 146 paired lesions (brain metastasis and primary tumor) from 73 NSCLC patients, PD-L1 expression (clone E1L3N) was reported in only one-third of brain metastases samples $(\mathrm{N}=24)$ and this percentage was numerically lower than that reported for the primary tumour $(\mathrm{N}=32,44 \% \mathrm{PD}$ L1 positive). Of note, $\mathrm{PD}-\mathrm{L} 1$ discordance between brain metastases and primary was higher if time elapsed between both samples is $\geq 6$ months ( $11 \%$ discordance versus $3 \%$ discordance if obtained $<6$ months apart, but the difference was not statistically significant) (13). T-cells can cross the blood-brain and blood tumor barrier, and can interact with brain metastases (14-16). It has been shown that the extent of T-cell infiltration correlates with survival in brain metastases patients, independently from other prognostic factors (16). Importantly, T-cell infiltration was not associated with $\mathrm{PD}$ L1 expression (16). Tumour mutational burden (TMB) has been suggested as another predictive marker for ICI benefit in NSCLC (17). The TMB is higher in brain metastases than in extracranial lesions. In a study with 13 paired samples, median TMB (calculated as the number of mutations (Mut) per megabase (Mb) of sequenced region after filtering, determined with whole exosome sequencing) was 24.9 Mut/Mb in brain metastases versus 12.5 Mut/Mb in the primary $(\mathrm{P}<0.0001)(18)$. In another study, including lung adenocarcinomas, median TMB (determined with a custom designed 592-gene next generation sequencing panel) was $13 \mathrm{Mut} / \mathrm{Mb}$ in brain metastases and 6 Mut/ $\mathrm{Mb}$ in the primary. Indeed, TMB in this study was a sitespecific biomarker with important spatial differences, and high TMB (defined as $\geq 10 \mathrm{Mut} / \mathrm{Mb}$ ) was more common in brain metastases compared with other metastatic sites (61\% vs. $35 \%$ for other metastases; $\mathrm{P}<0.001)$ or compared with primary adenocarcinoma $(61 \%$ vs. $25 \% ; \mathrm{P}<0.001)$. However, although brain metastases may express PD-L1 in $30 \%$ of cases and have a higher TMB, they also have an immune ignorant phenotype (e.g., lower T-cell infiltration, elevated tumor-associated macrophage infiltration, genes inhibiting dendritic cell maturation, TH-1 and leukocyte extravasation pathways, suppression of vascular cell adhesion protein 1) and a contraction in the number of $\mathrm{T}$-cell clones compared with the extracranial disease sites $(64 \%$ in brain metastases versus the primary tumor) $(13,18,19)$, which could potentially limit the intracranial ICI efficacy. This immune ignorant environment could in part explain the rate of dissociated intracranial and extracranial responses (i.e., progression in the brain and response extracranial or vice versa) reported in phase II and retrospective series, which ranges from $13 \%$ to $22 \%(9,10)$. For example, in the phase II study, six out of the 27 patients that were evaluable for both brain and systemic response had a dissociated response. Of note, three of these patients had a brain metastases response while progressing extracranially, while the other three experienced the opposite scenario (10). Percentage of dissociated responses in extracranial lesions only (e.g., enlargement of adrenal mass while response in pulmonary lesion) seems lower, as retrospective series reported percentages around $8 \%(20,21)$. Because of the retrospective nature of these analyses, no firm conclusions can be drawn.

\section{Monotherapy ICI data}

\section{Trial data}

First-line treatment with pembrolizumab monotherapy is 
advised for NSCLC patients with PD-L1 expression $\geq 50 \%$ (European Union, EU) or $\geq 1 \%$ (United States, USA), without a targetable driver alteration. In a recent pooled analysis $(\mathrm{N}=3,170)$ based on four KEYNOTE studies (001, 010, 024 and 42) including a total of 292 PD-L $1 \geq 1 \%$ NSCLC patients with brain metastases, the median OS with pembrolizumab was also superior to chemotherapy in the brain metastases subgroup: 13.4 versus 10.3 months [hazard ratio (HR) 0.83 ; $95 \%$ confidence interval (CI), $0.62-1.10]$. In the subgroup of NSCLC patients with high PD-L1 expression and brain metastases $(\mathrm{N}=159)$ the magnitude of benefit with pembrolizumab compared with chemotherapy increased, reaching a HR of 0.78 (95\% CI, 0.71-0.85). Importantly, both the magnitude of benefit with pembrolizumab and the toxicity profile were similar to those in patients without brain metastases (8), however, neurological toxicity was not specifically reported.

Atezolizumab is registered in the EU and US for second-line treatment of NSCLC patients based on the OAK trial $(22,23)$ as monotherapy and in combination with chemotherapy with or without bevacizumab in the first-line setting based on the IMPOWER 130 and IMPOWER 150 trial $(24,25)$. Patients with asymptomatic and treated brain metastases were eligible for the phase III OAK trial testing atezolizumab versus docetaxel. In an exploratory analysis, 123 out of the 850 patients (14\%) had brain metastases, and these patients also seemed to obtain benefit from atezolizumab. There was a trend towards longer survival compared with docetaxel: 16.0 vs. 11.9 months, HR 0.74; 95\% CI, 0.49-1.13. Likewise, time to development of brain metastases was delayed with atezolizumab. In the subgroup of patients with a history of asymptomatic treated baseline brain metastases, median time to the development of brain metastases was not reached in the atezolizumab arm, and was 9.3 months in the docetaxel arm (HR 0.38; 95\% CI, 0.16-0.91, P=0.0239) In those without baseline brain metastases, time to development of brain metastases was similar and not reached in both arms (HR 0.99; $95 \%$ CI, 0.50-1.97, $\mathrm{P}=0.9803$ ) (26). Similar results were found in the phase II FIR study (atezolizumab for advanced NSCLC patients selected based on PD-L1 expression). In cohort 3 , assessing the role of atezolizumab as second line or beyond, patients with treated brain metastases were included. Thirteen patients were evaluable for response. Investigator-assessed ORR was $23 \%$ in this cohort, with a median OS of 6.8 months, and 30-month OS rate of $19 \%$. Safety was similar to those without brain metastases, with $15 \%$ of grade 3-4 adverse events (AEs) (27). Despite the promising activity, these populations do not reflect patients managed in daily practice since patients with brain metastases were still highly selected. Unstable or untreated brain metastases were excluded, and a corticosteroid dose of $>10 \mathrm{mg}$ prednisolone equivalent/day was not allowed. In contrast, approximately $30 \%$ of patients with brain metastases in daily practice receive steroids ( $2 / 3$ receiving $>10 \mathrm{mg}$ prednisolone equivalent/day) (9). Two key points arising from this bias in "over-selecting" the eligible population for phase III trials evaluating ICIs are firstly, that it may explain the outcome discrepancies with the real-world population with brain metastases treated with ICIs, and secondly, the efficacy of ICIs in this real world population is largely unknown.

\section{Retrospective series and expanded access data}

Some clinical series reporting ICI efficacy in NSCLC patients with brain metastases exist, but most are small and retrospective (6). One of the largest retrospective series reported to date consisted of 1,025 patients, including 255 with brain metastases (39\% active, 14\% symptomatic and $27 \%$ treated with corticosteroids). Intracranial ORR was $27.3 \%$ with ICI and interestingly, pseudoprogression occurred in only $1 \%$ of patients, while a dissociated cranial-extracranial response occurred in $13 \%$. Patients with active brain metastases had more often brain metastases progression on ICI compared with those with stable brain metastases $(54 \%$ vs. $30 \%, \mathrm{P}<0.001)$. In multivariate analysis, the presence of brain metastases was not associated with OS (HR 0.99), but corticosteroid use was associated with poor OS (HR 2.4). Improved OS was correlated with higher disease specific Graded Prognostic Assessment (ds-GPA) and stable brain metastases (HR 0.5 and 0.6 , respectively) (9).

Several nivolumab expanded access programs (EAPs) have reported outcomes for (selected) brain metastases patients. The largest series are reported here. In an Italian EAP cohort, 37 out of 371 squamous lung cancer patients $(10 \%)$ and 409 out of 1,588 non-squamous lung cancer patients $(26 \%)$ had brain metastases. For the squamous histological subgroup, the 1 -year OS rates were $39 \%$ for the overall population and $35 \%$ for the brain metastases subgroup (28). For non-squamous, the 1-year OS rates were $48 \%$ and $43 \%$, respectively (29). Data for the French EAP ( $\mathrm{N}=902,35 \%$ squamous, $65 \%$ non-squamous) have also been reported. One hundred ninety-seven patients had baseline brain metastases (22\%), and brain metastases 
were associated with worse OS in multivariate analysis (HR $1.37, \mathrm{P}=0.0007)$, but correction for use of corticosteroids was not performed (30). In contrast, in the Dutch EAP series [ $\mathrm{N}=2,302$ patients with follow-up data, of which 389 $(16.9 \%)$ with brain metastases], the presence of baseline brain metastases was not associated with worse OS (HR 1.17, $\mathrm{P}=0.07)(31)$.

\section{ICI-chemotherapy data}

Similarly, in the first-line setting the combination of ICI plus chemotherapy has become the standard of care regardless of PD-L1 expression and histology subtype $(24,25,32,33)$. These combinations improve the OS compared with chemotherapy in the whole population and in patients with brain metastases, as was shown in a pooled analysis of three KEYNOTE trials (021, 189 and 407). Out of 1,298 included patients, 171 (13\%) had baseline brain metastases. In contrast to other studies, patients with stable untreated brain metastases and a maximum diameter of $15 \mathrm{~mm}$ were also allowed in the KEYNOTE 189 and 407 , but the exact proportion of patients with stable untreated brain metastases enrolled into these trials was not reported. With a median follow-up of approximately 11 months, pembrolizumab-chemotherapy for patients with brain metastases achieved a longer median OS compared to chemotherapy (18.8 vs. 7.6 months, HR 0.48; 95\% CI, 0.32-0.70). Of note, treatment related adverse events (TRAEs) of grade 3-5 were slightly higher in the pembrolizumab-chemotherapy brain metastases subgroup versus the group without brain metastases $(59.8 \% \mathrm{vs}$. $50.5 \%)$, although neurological TRAEs were similar for both groups (32.4\% vs. $36.4 \%)$ (7).

\section{Future directions}

ICI monotherapy has some efficacy in selected NSCLC patients with brain metastases. It is possible that ICIchemotherapy regimens are more effective in the treatment of patients with brain metastases from NSCLC. However, the pooled KEYNOTE analysis for the ICI-chemotherapy combination did not provide outcomes according to PDL1 strata; furthermore it remains unknown whether the pembrolizumab-chemotherapy combination is superior to pembrolizumab monotherapy in patients with brain metastases and PD-L 1 expression $\geq 50 \%$, since the combination could minimize the risk of symptomatic brain metastases progression. Moreover, comparisons between
ICI-chemotherapy combinations and ICI monotherapy are hampered, as different brain metastases groups were included. The phase II ATEZO-brain study (NCT03526900) is prospectively assessing the role of atezolizumab and carboplatin plus pemetrexed in chemotherapy-naïve patients with asymptomatic brain metastases.

Finally, data of these trials do not elucidate the role of PD-L1 as predictive marker for intracranial ICI efficacy. So, future challenges are relevant about how to improve outcomes for NSCLC patients with brain metastases.

Bevacizumab combined with chemotherapy resulted in an icORR of $61 \%$ in 67 advanced NSCLC patients with asymptomatic brain metastases (34). Indeed, bevacizumab prevents brain metastases development in preclinical studies and retrospective series (35), and acts synergistically with ICI (25). Furthermore, bevacizumab is effective in the treatment of radiation necrosis and can reduce the need for corticosteroids in this setting (36). This makes the combination of bevacizumab plus chemotherapy-ICI in brain metastases patients attractive. Unfortunately, brain metastases subgroup results have not been reported for the IMPOWER150 trial that assessed the quadruplet regimen carboplatin-paclitaxel-bevacizumab-atezolizumab (25). A trial evaluating pembrolizumab-bevacizumab is ongoing (NCT02681549). Another option is a combination of anti-PD(L)1 and anti-cytotoxic T-lymphocyte-associated protein4 (CTLA4) therapy, to hopefully increase T-cell infiltration and reduce regulatory T-cells (37). Evidence from this combination comes from melanoma patients with brain metastases: icORR was more than doubled in the nivolumab-ipilimumab treated patients compared with the nivolumab monotherapy treated patients (intracranial ORR $46 \%$ vs. $20 \%$ ) (38). For NSCLC, the only data available come from the CheckMate 817 trial. One arm included NSCLC special populations patients ( $\mathrm{N}=198)$, including asymptomatic untreated brain metastases patients with a good performance status $(\mathrm{N}=50,25 \%)$. For the special populations arm, median progression free survival (PFS) was 3.9 months (95\% CI, 2.8-5.4), median duration of response (DoR) was 13.8 months (9.6-not reached), and one-year DoR was $57 \%$ (39). In the final OS analysis, median OS was 9.9 months (95\% CI, 7.0-13.7) (40). Brain metastases specific outcomes have not been reported yet. In the CheckMate 227 trial (nivolumab-ipilimumab vs. chemotherapy), patients with treated brain metastases were eligible but outcomes for this subgroup have not been reported (41). Nivolumab-ipilimumab combined with SRT is currently under investigation (NCT 02696993). 
As radiotherapy can cause an abscopal effect in ICI treated patients, acts synergistically with ICI (42) and controls brain metastases, the added value of cranial radiotherapy to ICI is still under evaluation. Retrospective series suggest that stereotactic radiotherapy concurrent with ICI can improve survival, compared with sequential ICI-radiotherapy or radiotherapy-ICI $(6,43,44)$. These series have been extensively summarized in the review of $\mathrm{Li}$ et al. (44), and the international meta-analysis of individual patient data of Lehrer et al. (43). Of note, selection of patients for the sequence of treatments (upfront ICI followed by radiotherapy, upfront radiotherapy followed by ICI, ICI-radiotherapy concurrently) can be biased. For example, a neurologically symptomatic patient will start with upfront radiotherapy. Therefore, prospective randomized trials are urgently needed. Based on these series, it seems that PD-(L) 1 inhibitors can be safely combined with cranial irradiation, but detailed neurotoxicity assessments were not collected or provided. Furthermore, the incidence of radionecrosis does not seem to increase with $\mathrm{PD}-(\mathrm{L}) 1$ inhibitors, whereas it increases with anti-CTLA4 therapy $(6,43)$. Several ICI-radiotherapy trials are ongoing (e.g., NCT02858869, NCT02696993, NCT02978404, NCT02696993, NCT03955198) and results are awaited.

Moreover, the interpretation of brain imaging can be challenging in the context of ICI with or without cranial radiotherapy, as ICI can cause pseudoprogression of the brain metastases (45) and radiotherapy can cause both radiation necrosis and pseudoprogression (36). The immunotherapy Response Assessment for NeuroOncology (iRANO) advises to discontinue ICI in patients with progressing brain metastases that also clinically deteriorate regardless of timing, or in case of progression on imaging when a patient has been treated for more than 6 months with ICI. Continuation of ICI is advised in those progressing on imaging, but who are clinically stable and are treated for 6 months or less with ICI, with a repeat imaging 3 months after initial imaging. If this imaging confirms the progression, the patient should discontinue the ICI (46). However, applicability of iRANO outside of clinical trials remains a challenge and the best method for response assessment still needs to be defined. Positron emission tomography with radiolabelled amino acids such as ${ }^{18} \mathrm{~F}$-fluoroethyl-L-tyrosine, ${ }^{18} \mathrm{~F}$-fluorothymidine and ${ }^{11} \mathrm{C}$-methyl-L-methionine could be useful in the differentiation of radiation necrosis, pseudoprogression and real brain metastases progression. Furthermore, advanced magnetic resonance imaging (MRI) techniques such as perfusion-weighted imaging and diffusion-weighted imaging are under evaluation in brain metastases patients treated with ICI (47). Feroxymustil as a contrast agent is also under investigation in a phase II trial, to evaluate whether this contrast agent can differentiate between progression and pseudoprogression in NSCLC patients treated with pembrolizumab and stereotactic radiosurgery for the brain metastases (NCT03325166). The optimal tool for assessing the response as well as the applicability of dynamic radiological assessments for defining progression and toxicity are relevant challenges as number of patients with brain metastases who potentially may receive ICI in daily clinical practice is increasing as chemotherapy-ICI also has become an option.

Last, trials including patients with untreated brain metastases should foresee brain metastases related secondary or exploratory endpoints and bicompartmental response assessments (separate cranial and extracranial endpoints) as recommended by the Response Assessment in NeuroOncology Brain Metastases working group (48). They also advise to specify actions that are allowed if progression occurs in one compartment (e.g., stereotactic radiotherapy for a growing brain metastasis while continuing the ICI because of extracranial disease control).

\section{Conclusions}

In conclusion, selected NSCLC patients with brain metastases (asymptomatic and treated patients, or asymptomatic and small lesions) also benefit from ICI with or without chemotherapy. However, the best treatment strategy (ICI monotherapy vs. ICI-chemotherapy, or even ICI-ICI combinations, the addition of angiogenesis inhibition, ICI concurrent with cranial radiotherapy) still needs to be defined. Furthermore, the use of prognostic scores for brain metastases patients such as the disease specific Graded Prognostic Assessment needs to be validated in the immune-oncology era. Finally, based on the potential prolonged OS with ICI treatment, other factors become important. These are for example the risk of delayed neurotoxicity, incidence of radionecrosis and differentiation with brain metastases progression. These additional challenges urgently need to be addressed in daily clinical practice with detailed neurotoxicity assessments and new imaging techniques. 


\section{Acknowledgments}

Funding: None.

\section{Footnote}

Provenance and Peer Review: This article was commissioned by the Guest Editors (Jordi Remon and Benjamin Besse) for the focused issue "Immunotherapy in other thoracic malignancies and uncommon populations" published in Translational Lung Cancer Research. The article has undergone external peer review.

Conflicts of Interest: All authors have completed the ICMJE uniform disclosure form (available at http:// dx.doi.org/10.21037/tlcr-20-343). The focused issue "Immunotherapy in other thoracic malignancies and uncommon populations" was commissioned by the editorial office without any funding or sponsorship. JR served as the unpaid Guest Editor of the focused issue and serves as an unpaid editorial board member of Translational Lung Cancer Research from Sep 2019 to Sep 2021. BB served as the unpaid Guest Editor of the focused issue. LH: none related to current manuscript, outside of current manuscript: research funding Roche, Boehringer Ingelheim, AstraZeneca (all institution), advisory board: Boehringer, BMS, Lilly, Roche, Pfizer, Takeda, MSD (all institution), speaker: MSD, travel/ conference reimbursement: Roche, BMS (self); mentorship program with key opinion leaders: funded by AstraZeneca; fees for educational webinars: Quadia (self), interview sessions funded by Roche (institution). JR: none related to current manuscript, outside of current manuscript: advisory: Boehringer-Ingelheim, MSD, Pfizer, BMS, AstraZeneca. Travel: OSE immunotherapeutics, BMS, AstraZeneca. JM: none related to current manuscript, outside of current manuscript: advisory: Boehringer-Ingelheim, MSD, Roche. Travel: Boehringer-Ingelheim, MSD, BMS, AstraZeneca. BB: Sponsored Research at Gustave Roussy Cancer Center Abbvie, Amgen, AstraZeneca, Biogen, Blueprint Medicines, BMS, Celgene, Eli Lilly, GSK, Ignyta, IPSEN, Merck KGaA, MSD, Nektar, Onxeo, Pfizer, Pharma Mar, Sanofi, Spectrum Pharmaceuticals, Takeda, Tiziana Pharma. The authors have no other conflicts of interest to declare.

Ethical Statement: The authors are accountable for all aspects of the work in ensuring that questions related to the accuracy or integrity of any part of the work are appropriately investigated and resolved.
Open Access Statement: This is an Open Access article distributed in accordance with the Creative Commons Attribution-NonCommercial-NoDerivs 4.0 International License (CC BY-NC-ND 4.0), which permits the noncommercial replication and distribution of the article with the strict proviso that no changes or edits are made and the original work is properly cited (including links to both the formal publication through the relevant DOI and the license). See: https://creativecommons.org/licenses/by-ncnd $/ 4.0 /$

\section{References}

1. Peters S, Bexelius C, Munk V, et al. The impact of brain metastasis on quality of life, resource utilization and survival in patients with non-small-cell lung cancer. Cancer Treat Rev 2016;45:139-62.

2. Huber RM, Hansen KH, Paz-Ares Rodriguez L, et al. Brigatinib in Crizotinib-Refractory ALK+ Non-Small Cell Lung Cancer: 2-Year Follow-up on Systemic and Intracranial Outcomes in the Phase 2 ALTA Trial. J Thorac Oncol 2020;15:404-15.

3. Rangachari D, Yamaguchi N, VanderLaan PA, et al. Brain metastases in patients with EGFR-mutated or ALKrearranged non-small-cell lung cancers. Lung Cancer 2015;88:108-11.

4. Berghoff AS, Schur S, Fureder LM, et al. Descriptive statistical analysis of a real life cohort of 2419 patients with brain metastases of solid cancers. ESMO Open 2016;1:e000024.

5. Garon EB, Hellmann MD, Rizvi NA, et al. Five-Year Overall Survival for Patients With Advanced NonSmallCell Lung Cancer Treated With Pembrolizumab: Results From the Phase I KEYNOTE-001 Study. J Clin Oncol 2019;37:2518-27.

6. El Rassy E, Botticella A, Kattan J, et al. Non-small cell lung cancer brain metastases and the immune system: From brain metastases development to treatment. Cancer Treat Rev 2018;68:69-79.

7. Powell SF, Rodrigeuz-abreu D, Langer C, et al. Pembrolizumab Plus Platinum Based Chemotherapy in NSCLC With Brain Metastases: Pooled Analysis of KEYNOTE 021,189 , and 407. Ann Oncol 2019;30:v602-v60.

8. Mansfield A, Herbst R, Castro G, et al. Outcomes With Pembrolizumab Monotherapy in Patients With PD L1 Positive NSCLC With Brain Metastases: Pooled Analysis of KEYNOTE 001, 010, 024, and 042. Ann Oncol 
2019;30:v602-v60.

9. Hendriks LEL, Henon C, Auclin E, et al. Outcome of Patients with Non-Small Cell Lung Cancer and Brain Metastases Treated with Checkpoint Inhibitors. J Thorac Oncol 2019;14:1244-54.

10. Goldberg SB, Schalper KA, Gettinger SN, et al. Pembrolizumab for management of patients with NSCLC and brain metastases: long-term results and biomarker analysis from a non-randomised, open-label, phase 2 trial. Lancet Oncol 2020;21:655-63.

11. Goldberg S, Gettinger S, Mahajan A, et al. Durability of brain metastasis response and overall survival in patients with non-small cell lung cancer (NSCLC) treated with pembrolizumab. J Clin Oncol 2018;36:abstr 2009.

12. Proto C, Ferrara R, Signorelli D, et al. Choosing wisely first line immunotherapy in non-small cell lung cancer (NSCLC): what to add and what to leave out. Cancer Treat Rev 2019;75:39-51.

13. Mansfield AS, Aubry MC, Moser JC, et al. Temporal and spatial discordance of programmed cell death-ligand 1 expression and lymphocyte tumor infiltration between paired primary lesions and brain metastases in lung cancer. Ann Oncol 2016;27:1953-8.

14. Ransohoff RM, Engelhardt B. The anatomical and cellular basis of immune surveillance in the central nervous system. Nat Rev Immunol 2012;12:623-35.

15. Berghoff AS, Preusser M. The inflammatory microenvironment in brain metastases: potential treatment target? Chin Clin Oncol 2015;4:21.

16. Berghoff AS, Fuchs E, Ricken G, et al. Density of tumorinfiltrating lymphocytes correlates with extent of brain edema and overall survival time in patients with brain metastases. Oncoimmunology 2016;5:e1057388.

17. Hendriks LE, Rouleau E, Besse B. Clinical utility of tumor mutational burden in patients with non-small cell lung cancer treated with immunotherapy. Transl Lung Cancer Res 2018;7:647-60.

18. Mansfield AS, Ren H, Sutor S, et al. Contraction of T cell richness in lung cancer brain metastases. Sci Rep 2018;8:2171.

19. Kudo Y, Haymaker C, Zhang J, et al. Suppressed immune microenvironment and repertoire in brain metastases from patients with resected non-small-cell lung cancer. Ann Oncol 2019;30:1521-30.

20. Tazdait M, Mezquita L, Lahmar J, et al. Patterns of responses in metastatic NSCLC during PD-1 or PDL-1 inhibitor therapy: Comparison of RECIST 1.1, irRECIST and iRECIST criteria. Eur J Cancer 2018;88:38-47.
21. Vaflard P, Paoletti X, Servois V, et al. Dissociated responses in patients with metastatic solid tumors treated with immunotherapy. Ann Oncol 2019;30:v475-v532.

22. Rittmeyer A, Barlesi F, Waterkamp D, et al. Atezolizumab versus docetaxel in patients with previously treated non-small-cell lung cancer (OAK): a phase 3, openlabel, multicentre randomised controlled trial. Lancet 2017;389:255-65.

23. Fehrenbacher L, von Pawel J, Park K, et al. Updated Efficacy Analysis Including Secondary Population Results for OAK: A Randomized Phase III Study of Atezolizumab versus Docetaxel in Patients with Previously Treated Advanced Non-Small Cell Lung Cancer. J Thorac Oncol 2018;13:1156-70.

24. West H, McCleod M, Hussein M, et al. Atezolizumab in combination with carboplatin plus nab-paclitaxel chemotherapy compared with chemotherapy alone as firstline treatment for metastatic non-squamous non-smallcell lung cancer (IMpower130): a multicentre, randomised, open-label, phase 3 trial. Lancet Oncol 2019;20:924-37.

25. Socinski MA, Jotte RM, Cappuzzo F, et al. Atezolizumab for First-Line Treatment of Metastatic Nonsquamous NSCLC. N Engl J Med 2018;378:2288-301.

26. Gadgeel SM, Lukas RV, Goldschmidt J, et al. Atezolizumab in patients with advanced non-small cell lung cancer and history of asymptomatic, treated brain metastases: Exploratory analyses of the phase III OAK study. Lung Cancer 2019;128:105-12.

27. Spigel DR, Chaft JE, Gettinger S, et al. FIR: Efficacy, Safety, and Biomarker Analysis of a Phase II Open-Label Study of Atezolizumab in PD-L1-Selected Patients With NSCLC. J Thorac Oncol 2018;13:1733-42.

28. Cortinovis D, Chiari R, Catino A, et al. Italian Cohort of the Nivolumab EAP in Squamous NSCLC: Efficacy and Safety in Patients With CNS Metastases. Anticancer Res 2019;39:4265-71.

29. Crino L, Bronte G, Bidoli P, et al. Nivolumab and brain metastases in patients with advanced non-squamous nonsmall cell lung cancer. Lung Cancer 2019;129:35-40.

30. Molinier O, Audigier-Valette C, Cadranel J, et al. IFCT1502 CLINIVO: Real-life experience with nivolumab in patients with advanced NSCLC. J Thorac Oncol 2017;12:1793.

31. Smit HJM, Aerts J, van den Heuvel M, et al. Effects of checkpoint inhibitors in advanced non-small cell lung cancer at population level from the National Immunotherapy Registry. Lung Cancer 2020;140:107-12. 32. Gandhi L, Rodriguez-Abreu D, Gadgeel S, et al. 
Pembrolizumab plus Chemotherapy in Metastatic NonSmall-Cell Lung Cancer. N Engl J Med 2018;378:2078-92.

33. Paz-Ares L, Luft A, Vicente D, et al. Pembrolizumab plus Chemotherapy for Squamous Non-Small-Cell Lung Cancer. N Engl J Med 2018;379:2040-51.

34. Besse B, Le Moulec S, Mazieres J, et al. Bevacizumab in Patients with Nonsquamous Non-Small Cell Lung Cancer and Asymptomatic, Untreated Brain Metastases (BRAIN): A Nonrandomized, Phase II Study. Clin Cancer Res 2015;21:1896-903.

35. Ilhan-Mutlu A, Osswald M, Liao Y, et al. Bevacizumab prevents brain metastases formation in lung adenocarcinoma. Mol Cancer Ther 2016;15:702-10.

36. Loganadane G, Dhermain F, Louvel G, et al. Brain Radiation Necrosis: Current Management With a Focus on Non-small Cell Lung Cancer Patients. Front Oncol 2018;8:336.

37. Spranger S, Koblish HK, Horton B, et al. Mechanism of tumor rejection with doublets of CTLA-4, PD-1/PD-L1, or IDO blockade involves restored IL-2 production and proliferation of CD8(+) T cells directly within the tumor microenvironment. J Immunother Cancer 2014;2:3.

38. Long GV, Atkinson V, Lo S, et al. Combination nivolumab and ipilimumab or nivolumab alone in melanoma brain metastases: a multicentre randomised phase 2 study. Lancet Oncol 2018;19:672-81.

39. Barlesi F, Audigier-Valette C, E. F, et al. OA04.02 CheckMate 817: First-Line Nivolumab + Ipilimumab in Patients with ECOG PS 2 and Other Special Populations with Advanced NSCLC. J Thorac Oncol 2019;14:S214-5.

40. Barlesi F, Audigier-Valette C, Felip E, et al. Nivolumab plus low-dose ipilimumab as first-line treatment of advanced NSCLC: Overall survival analysis of checkmate
817. Ann Oncol 2019;30:xi33-xi47.

41. Hellmann MD, Ciuleanu TE, Pluzanski A, et al. Nivolumab plus Ipilimumab in Lung Cancer with a High Tumor Mutational Burden. N Engl J Med 2018;378:2093-104.

42. Ishihara D, Pop L, Takeshima T, et al. Rationale and evidence to combine radiation therapy and immunotherapy for cancer treatment. Cancer Immunol Immunother 2017;66:281-98.

43. Lehrer EJ, Peterson J, Brown PD, et al. Treatment of brain metastases with stereotactic radiosurgery and immune checkpoint inhibitors: An international meta-analysis of individual patient data. Radiother Oncol 2019;130:104-12.

44. Li W, Yu H. Separating or combining immune checkpoint inhibitors (ICIs) and radiotherapy in the treatment of NSCLC brain metastases. J Cancer Res Clin Oncol 2020;146:137-52.

45. Doherty MK, Jao K, Shepherd FA, et al. Central Nervous System Pseudoprogression in a Patient Treated with PD-1 Checkpoint Inhibitor. J Thorac Oncol 2015;10:e100-1.

46. Okada H, Weller M, Huang R, et al. Immunotherapy response assessment in neuro-oncology: a report of the RANO working group. Lancet Oncol 2015;16:e534-42.

47. Galldiks N, Lohmann P, Werner JM, et al. Molecular imaging and advanced MRI findings following immunotherapy in patients with brain tumors. Expert Rev Anticancer Ther 2020;20:9-15.

48. Camidge DR, Lee EQ, Lin NU, et al. Clinical trial design for systemic agents in patients with brain metastases from solid tumours: a guideline by the Response Assessment in Neuro-Oncology Brain Metastases working group. Lancet Oncol 2018;19:e20-e32.
Cite this article as: Hendriks LEL, Remon J, Menis J, Besse B. Is there any opportunity for immune checkpoint inhibitor therapy in non-small cell lung cancer patients with brain metastases? Transl Lung Cancer Res 2021;10(6):2868-2875. doi: $10.21037 /$ tlcr-20-343 